\title{
Life Threatening Zygomyces Infection Of The Gastrointestinal Tract
}

\author{
Mohanad Al-Qaisi, MD ${ }^{1}$ \\ Charles Stauffer, $M D^{1}$ \\ Gerges Makar, $M D^{1}$ \\ Tim Kuberski, $M D^{2}$ \\ ${ }^{1}$ Department of Medicine, Maricopa Medical Center, Phoenix, Arizona \\ ${ }^{2}$ Department of Medicine, Infectious Diseases, Maricopa Medical Center, \\ Phoenix, Arizona
}

\begin{abstract}
A 25 year old diabetic woman was admitted into the Intensive Care Unit because of ketoacidosis, hypotension and upper gastrointestinal bleeding. Emergency endoscopic biopsy of the upper gastrointestinal tract demonstrated invasive, non-septate fungal hyphae suggestive of either a Zygomyces or Basidiobolus. Amphotericin B was not used because of its ineffectiveness against Basidiobolus and her renal failure. In addition, first generation antifungal azoles were not used because of their ineffectiveness against Zygomyces. The patient responded to medical therapy and the broad-spectrum azole antifungal posaconazole which has activity against both Basidiobolus and Zygomyces. The patient recovered from her critical illness and on follow up was without residual problems.
\end{abstract}

\section{Introduction}

Zygomyces are a group of fungi which include Mucor, Rhizopus and Absidia, the more common pathologic fungi in the order of Mucorales.

As a group, these fungi are characterized by having non-septate hyphae and cause aggressive angioinvasion in certain immunosuppressed settings like ketoacidosis (1). We present a patient who presented with a life-threatening septic syndrome, ketoacidosis and gastrointestinal bleeding due to an infection by an unknown non-septate hyphal fungus, eventually identified as Rhizopus species. On presentation the patient was critically ill and admitted to the Intensive Care Unit. Her early course was complicated by a therapeutic antifungal dilemma which could influence her survival.

\section{Case Report}

A 25 years old woman with diabetes was admitted to the Intensive Care Unit with septic syndrome and diabetic ketoacidosis. She was hypotensive, blood pressure was $75 / 42 \mathrm{mmHg}$, heart rate $147 / \mathrm{min}$, temperature $38.7 \mathrm{C}$. Pertinent blood testing revealed the following; glucose $623 \mathrm{mg} / \mathrm{dl}$, creatinine $2.15 \mathrm{mg} / \mathrm{dl}$, 
bicarbonate $13.6 \mathrm{mmol} / \mathrm{L}$, lactic acid $6.8 \mathrm{mmol} / \mathrm{L}, \mathrm{WBC} 9200 / \mu \mathrm{L}$, hemoglobin $7.4 \mathrm{gm} / \mathrm{dl}$. She was treated aggressively with intubation, mechanical ventilation, vasopressors and continuous renal replacement therapy (CRRT). Her diabetes was treated conventionally. Her course was complicated by a drop in hemoglobin from 11.4 to $7.0 \mathrm{gm} / \mathrm{dl}$ despite transfusions. Her stool was found to be hemoccult positive.

Upper endoscopy showed multiple ulcers involving the gastric body extending onto the cardia which was covered with coffee ground exudate. Biopsies were obtained and showed a "fungus" with non-septate hyphae on preliminary histopathology and amphotericin B was initiated empirically. She continued to experience significant hematemesis and hypotension requiring multiple transfusions (4 units). The amphotericin B was discontinued because of progressive azotemia. Upon review of the pathology from the stomach biopsy, the possibility was raised that she might have either a Basidiobolus or Zygomyces infection (Figure 1).

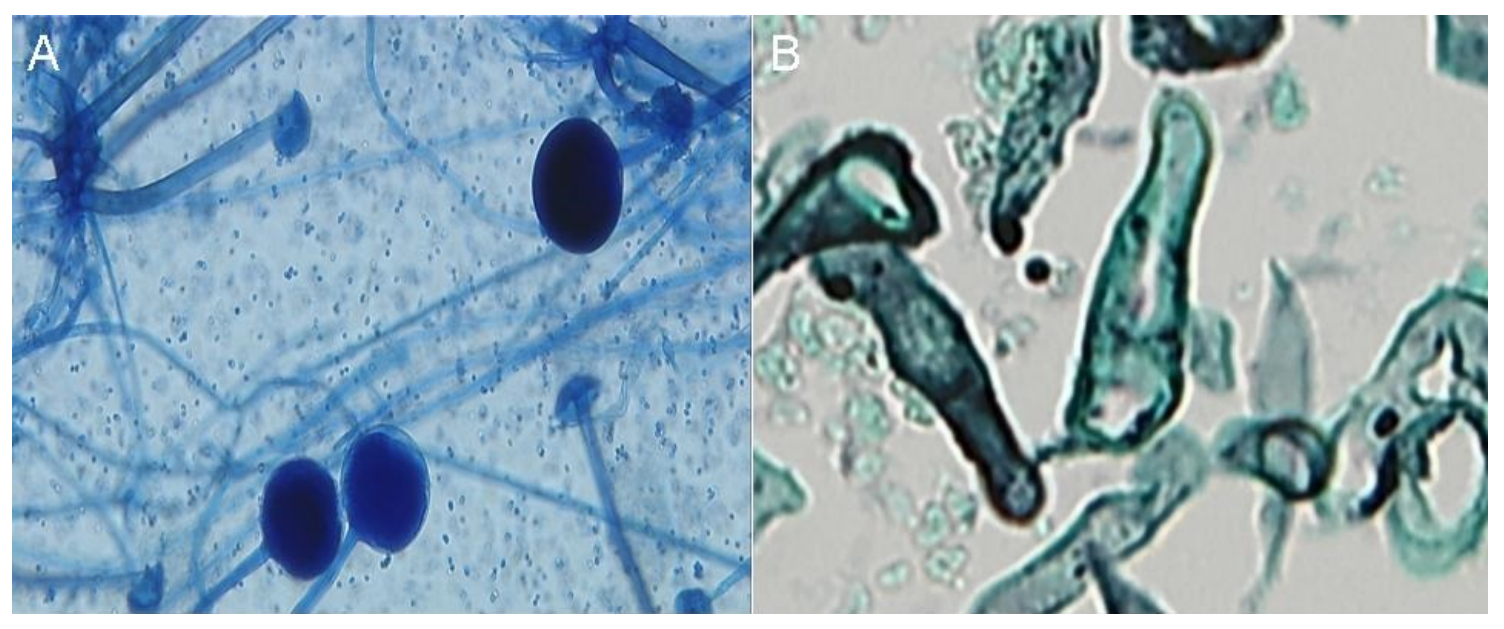

Figure 1 illustrates the difference in appearance of non-septate hyphae between fungi in tissue in vivo and on culture in vitro. (A) Fungal culture (in vitro) showing the non- septate hyphae. (B) GMS stain of the stomach tissue from the patient (in vivo) showing fungus fragments having broad, irregular, non-septate hyphae, arrow. The hyphae morphology becomes distorted with angioinvasion and tissue necrosis.

The patient was started empirically on oral posaconazole $400 \mathrm{mg}$ twice daily which theoretically would be effective for both fungi. Within a few days the ketoacidosis resolved, the gastrointestinal bleeding stopped and she was discharged a few days later. After her discharge a Rhizopus species was cultured and identified as the causative agent. 


\section{Discussion}

The therapeutic dilemma in the treatment of this patient was related to the inability to differentiate between two potential fungal pathogens, Zygomyces or Basidiobolus on the basis of only tissue pathology. Under ideal circumstances the histopathology might differentiate the two, however trying to distinguish between the two can be difficult because both have non-septate hyphae, are morphologically similar, and can involve the stomach. Based on morphology the differentiation between Basidiobolus and Rhizopus is subtle. For Basidiobolus the hyphal elements typically show "sparse" septation while Rhizopus hyphal elements show "infrequent" septation. There was an added problem in that confirmatory cultures can take weeks before a specific identification can be made. Zygomyces infections tend to be rapidly destructive, but are rare to involve the gastrointestinal tract (1). In contrast, Basidiobolus rananum is endemic to Arizona and generally known to primarily cause gastrointestinal infections (2). That organism however, usually causes an indolent process and is less likely to be fatal. However, there is a case report of angioinvasive disease with basidiobolomycosis reminiscent of mucormycosis in diabetics (3). Epidemiological studies on Basidiobolus suggest that the common risks for this infection include living in Arizona, having diabetes and use of medications that suppress stomach acids (2).

A high index of suspicion in our patient with some of these risk factors made Basidiobolus a consideration. Importantly, the antifungal treatment of Basidiobolus is different than for the Zygomyces (i.e., Rhizopus). Basidiobolus is known to be resistant to amphotericin $B$ and the preferred treatment is itraconazole (2).

Our patient initially received a few doses of amphotericin B empirically because of the report of a non-septate "fungus" on biopsy. Amphotericin B is the drug of choice for Zygomyces, but not for Basidiobolus (4). Notably itraconazole is not effective for the Zygomyces. The treatment decision was made to use posaconazole because of its broad spectrum antifungal activity that would have activity against both Zygomyces and Basidiobolus. In addition, there is a report of posaconazole being used successfully to treat gastrointestinal basidiobolomycosis (5). Of the Zygomyces, Rhizopus is the most common cause of human infections, more than Mucor. Certainly correcting the ketoacidosis and gastrointestinal bleeding contributed to her improvement, but the mortality rate in diabetic patients with Zygomyces involving the gastrointestinal tract is about $85 \%(6)$. The patient appeared to be effectively treated based on the therapeutic antifungal decision while the patient was critically ill. She was seen in follow up several weeks later without any obvious residual effects. Her response to posaconazole suggests it would be an effective consideration in places like Arizona where Basidiobolus and Zygomyces could be in the differential. 


\section{References}

1. Chayakulkeeree M, Ghannoum MA, Perfect JR. Zygomycosis: the reemerging fungal infection. Eur J Clin Microbiol Infect Dis. 2006;25(4):21529. [CrossRef] [PubMed]

2. Vikram HR, Smilack JD, Leighton JA, Crowell MD, De Petris G.. Emergence of gastrointestinal basidiobolomycosis in the united states, with a review of worldwide cases. Clin Infect Dis. 2012;54(12):1685-91. [CrossRef] [PubMed]

3. Bigliazzi C, Poletti V, Dell'Amore D, Saragoni L, Colby TV. Disseminated basidiobolomycosis in an immunocompetent woman. J Clin Microbiol. 2004;42(3):1367-9. [CrossRef] [PubMed]

4. Guarro J, Aguilar C, Pujol I. In-vitro antifungal susceptibilities of Basidiobolus and Conidiobolus spp. strains. J Antimicrob Chemother. 1999;44(4):557-60. [CrossRefl [PubMed]

5. Rose RR, Lindsby MD, Hurst SF, Paddock CD, Damodaran T, Bennett J. Gastrointestinal basidiobolomycosis treated with posaconazole. Med Mycol Case Rep. 2012;2:11-4. [CrossRef] [PubMed]

6. Roden MM, Zaoutis TE, Buchanon WL, Knudsen TA, Sarkisova TA, Schaufele RL, Sein M, Sein T, Chiou CC, Chu JH, Kontoyiannis DP, Walsh JT. Epidemiology and outcome of zygomycosis: a review of 929 reported cases. Clin Infect Dis. 2005;41(5):634-53. [CrossRef] [PubMed] 